\title{
Controlling Crystal Growth with Modifiers
}

Franca Jones, ${ }^{a}$ and Mark I. Ogden ${ }^{a}$

\section{Received (in XXX, XXX) Xth XXXXXXXXX 200X, Accepted Xth XXXXXXXXX 200X}

First published on the web Xth XXXXXXXXX 200X

DOI: $10.1039 / \mathrm{b} 000000 x$

Modifying crystal growth processes using additives is a well established approach to solving problems in many processes. Nature also makes extensive use of crystalline inorganic structures modified by soluble and insoluble organic materials. This Highlight discusses some of the recent and interesting developments in this area, with an emphasis on the control of crystal growth rates, covering both inhibitors and the lesser known promoters of crystal growth. Hybrid materials resulting from the incorporation of modifiers into crystalline structures are also discussed, with an emphasis on nonclassical crystallisation mechanisms, involving the oriented attachment of nanoparticles.

\section{Introduction}

The field of crystallization is large and diverse, and only a small aspect can be covered in this Highlight. We will focus on crystals grown from solution, and the use of additives - inorganic and organic - to control or modify the crystal growth process. While this is a very well established area, with hugely important industrial applications (such as the control of scale in hydrometallurgy amongst others), recent developments show that there are still exciting opportunities to better understand the fundamental science of how additives can be used to control crystal growth. This is becoming ever more critical as nanotechnology requires a degree of control over crystallization as never before. ${ }^{1}$ As is so often the case, Nature has mastered crystal formation to a much greater extent than we humans and so, not surprisingly, crystal growth as biomineralization has been the focus of a vast literature which serves as an inspiration for much of the work that will be referred to here.

Crystal growth modifiers can be used to achieve a range of outcomes. They can dramatically affect particle shape and size and therefore can be used in a particle engineering sense, i.e. to obtain the desired physical properties of the particles in question. Additives can also inhibit nucleation and growth which has applications such as scale inhibition, for example, as mentioned above. They may even increase the rate of crystallization and be used as promoters. For the most part, the many studies of crystal growth modifiers interpret results using a thermodynamic approach only. There is good reason for this: measuring the kinetics of crystallization is much harder, especially when thermodynamic events (such as adsorption at growth features) can lead to kinetic impacts (slowing down of step growth). It is becoming increasingly clear, however, that kinetic factors are very important and need to be investigated.

Thus, there are still many fundamental questions to be answered about crystallization and the impact of modifiers before the ideal outcome is achieved - the a priori prediction of the effect of additives and their rational design for specific applications. This Highlight aims to review some of the recent developments that bring this aim closer.

\section{Crystal Growth Inhibition}

The introduction of additives to inhibit the nucleation and growth of crystalline materials has been well established for many years. Perhaps the most economically significant application of this is the reduction of scale formation in industrial processes. A classical example of this is heat exchangers where the inverse solubility behaviour of sparingly soluble salts such as gypsum causes supersaturation to increase when the solution is heated. Two classes of additives that have been widely used to inhibit 
scale formation are low molecular weight phosphonates, and polymeric carboxylates such as polyacrylates. The impact of polymers upon crystal growth mechanisms has been intensively investigated recently, and a number of reviews are available. ${ }^{2-4}$ Polymeric additives will be discussed below in the context of developments in the elucidation of crystal growth mechanisms. Some of the literature involving the study of the phosphonates will be discussed here as an example of typical approaches used in the study of crystal growth inhibitors.

The experimental observations of the impact of a phosphonate-based additive upon the crystal growth of an inorganic material such as barium sulfate typically include the reduction of the rate of nucleation and crystal growth, and very often a change in the crystal morphology and/or size. Generally speaking, the greater the concentration of the additive the smaller the particle size, ${ }^{5}$ however, there may be exceptions and it is best to investigate this explicitly. For example, as supersaturation increases particle size normally decreases but this does not occur with lactose. ${ }^{6}$ The changes in morphology can provide an indication as to which crystal faces are preferentially adsorbing the additive, as such faces will grow more slowly and become more dominant in the resulting morphology. A typical rationalization of the face-selective adsorption of the additive is "lattice matching", where the anionic moieties replace lattice anions at the crystal surface (see Figure 1). ${ }^{7}$ The incorporation of the impurity at the crystal surface then inhibits the further growth of the crystal surface. In order to lattice match the impurity need not be fully incorporated nor does it have to have the exact same spacing. In many instances, the impurity may only partly match the lattice spacing, but comparison of different additive structures relative to the crystal lattice does often provide some explanation of the relative efficacy of the additives. This is in essence a simplistic model involving in vacuo adsorption at a flat crystal surface but molecular modeling studies have shown good correlation with experimental results, provided factors such as the strain in the organic additive conformation is accounted for, particularly in small molecules. ${ }^{8,9}$

Fig. 1 In this image, the phosphonate groups $(\mathrm{P}=$ cream coloured spheres, $\mathrm{O}=$ red coloured spheres) on the EDTP molecule are able to lattice match the sulfate positions in the barium sulfate lattice. (Reproduced with permission from J. Phys. Chem. B, 2006, 110, 7414. Copyright 2006 American Chemical Society). ${ }^{8}$

It is likely that the success of simple models such as this depends on the dominance of electrostatic forces between the negatively charged additive and cations in the crystal lattice. Even so, there are often discrepancies between experimental results and modeling, and at that point additional factors, such as step and kink sites on the crystal surface, and/or solvation of the system, are often invoked. ${ }^{10}$ Perhaps the best indication that factors other than structural similarities between additive and crystal are involved in crystal growth modification, is the fact that this aspect is invoked in examples of both crystal growth inhibition, and promotion.

\section{Crystallisation Promotion}

Historically, much of the literature in this area has focused on the promotion of growing organic crystals. This is in part due to the fact that organic crystals (through pharmaceutical interests) have been more investigated. In addition, there has been traditionally more manipulation of their crystallization through use of anti solvents, co-solvents etc. The work of Weissbuch and colleagues, in particular, showed that the addition of similarly structured molecules could change the crystallization behaviour of the desired product. ${ }^{11}$ Thus, the socalled 'tailor-made' additive was coined. However, the organic molecule having a similar structural motif was used for explaining the results of both inhibition and promotion. Thus, it was not possible to predict if an additive was likely to be a promoter or an inhibitor. This is also, at a general level, what was claimed an important criteria for inhibiting inorganic crystallization through 'lattice matching' as discussed above. Thus, both the inhibitor molecule and the promoting molecule had similar 'features'. Clearly, this does not improve 
our understanding of why certain molecules have a particular impact on crystallization. More recently, the work of Volkmer and his group suggests that lattice matching is not important at all and non-specific electrostatic or dipole interactions are operating. ${ }^{12}$

There is evidence that promotion also occurs in inorganic systems. Some of the earliest work on an inorganic system was by Amsler in 1942 looking at the crystallization of potassium chloride in water and water/ethanol mixtures. ${ }^{13}$ Amsler's work used the change in conductivity to measure the induction times of the various potassium salts' crystallization and thereby obtained information on the critical nucleus size, but the same work clearly shows a shorter induction time for $\mathrm{KCl}$ in the presence of $25 \%$ ethanol even when solubility in the $25 \%$ ethanol/water mixture has been taken into consideration. Others such as Kalia, describe the promotion of $\mathrm{KHSO}_{4}$ crystallization in the presence of $\mathrm{Fe}^{3+}$ but give little mechanistic information. ${ }^{14}$

\section{Inorganic Promoters}

The work of Lui et al. showed that barite $\left(\mathrm{BaSO}_{4}\right)$ crystallized in the presence of sodium and potassium ions were rougher than those in their absence. ${ }^{15}$ This suggests that these ions promote barite crystallization. He et al. showed more specifically that singly charged cations lowered the induction time as concentration increased thus showing that nucleation was promoted in the presence of these singly charged ions; the larger the cation, the greater the impact. ${ }^{16}$ They hypothesised that an increase in solubility (and, therefore, a decrease in interfacial energy) was the cause. More recent work by Risthaus et al. ${ }^{17}$ and Kowacz and Putnis ${ }^{18}$ suggests, however, that the monocationic ions are not so much affecting the solubility but rather are changing the kinetics of barium sulfate crystallization. The work by Risthaus et al. ${ }^{17}$ and Becker et al. ${ }^{19}$ suggested the role of temporarily adsorbed singly charged cations lowering the activation energy for crystallization by the formation of a kink site. Over time, these monovalent cations would be replaced by $\mathrm{Ba}^{2+}$ (see Figure $2 \mathrm{a}$ ). In addition, it was experimentally observed that there was a clear difference in the growth island shape at higher ionic strength (see Figure 2b). The more recent work by Kowacz and Putnis, ${ }^{20}$ looks more deeply at the role of solvent as well as the monovalent cations on crystallization. They suggest that the ions impact in a 'Hofmeister series'-like manner and crystallization kinetics are altered depending on their impact on water structure. The impact on the crystallization promotion was found to be qualitatively predicted by their solubility. Similarly, Pina et $a l .{ }^{21}$ showed that even the simple case of solid solutions can be more complex than expected. While, thermodynamically, mixed $\mathrm{Sr} / \mathrm{BaSO}_{4}$ nuclei would be expected based on supersaturation arguments, $\mathrm{Sr}$ rich sulfate nuclei were observed due to the $\mathrm{SrSO}_{4}$ being kinetically favoured.

Fig. 2 (a) How sodium ions might lower the electrostatic barrier to $\mathrm{Ba}^{2+}$ incorporation, and (b) the effect of high ionic strength on growth spiral of barium sulfate. (Reproduced from Colloids Surf. A, 2001, 191, 201, Copyright (2001), with permission from Elsevier Science BV). ${ }^{17}$

Our own work has shown that barite crystallization, even in the absence of impurity ions, must be viewed both thermodynamically and kinetically to fully appreciate the impact impurities have. ${ }^{22-24}$ The thermodynamically stable faces are the $(010)$, the $(001)$ and the (210), yet in the absence of impurities the (010) face is almost never observed. Molecular dynamics modeling of these faces showed that the (010) face is self-cataysed by the presence of sulfate ions and that the rate determining step for the crystallization is the de-hydration of the barium ion. ${ }^{23}$ We decided to test this by undertaking crystallization in the presence of methanol and showed that even when the thermodynamic driving force (S) is equivalent the kinetics of crystallization are much greater in the presence of methanol (see Figure 3). ${ }^{22}$ The methanol changes the kinetic barrier of the barium ion desolvation and increases the (001) face growth rate in particular. Work by Kowacz 
et al., ${ }^{18}$ has supported these results showing that nucleation and growth is faster on the (001) face of barite even when the thermodynamic driving force is constant (see Figure 4).

Fig. 3 Barite morphology at $S=25$ in (a) pure water and (b) in $25 \mathrm{wt} \%$ methanol/water mix. (Reproduced with permission from Cryst. Growth Des., 2008, 8, 817. Copyright 2008 American Chemical Society). ${ }^{22}$

Fig. 4 Growth rate of the (001) face of barite in pure water and in water/methanol mix. (Reproduced from Geochim. Cosmochim. Acta, 2007, 71, 5168, Copyright (2007), with permission from Elsevier). ${ }^{18}$

We can also probe the kinetic effects morphologically. The DG of a reaction is expected to vary slightly with temperature, ${ }^{25}$. This is known as the "law of compensation" where the Gibbs energy will be little altered by small changes in temperature (since enthalpy and entropy both tend to alter in the same way increase or decrease - with temperature and their signs opposite). Thus, it is possible to increase the relative effect of kinetics on the crystallization process by increasing the temperature since the kinetics will increase due to the rate constant being proportional to $\mathrm{e}^{-(\mathrm{Ea} / \mathrm{RT})}$. However, in order to properly compare samples, the morphological changes as $\mathrm{S}$ varies for barium sulfate, must first be determined. Table 1 shows the morphology of barite at varying $\mathrm{S}$ levels at $75{ }^{\circ} \mathrm{C}$.

Table 1 Barium sulfate particles crystallized at $75{ }^{\circ} \mathrm{C}$ from $\mathrm{Na}_{2} \mathrm{SO}_{4}$ and $\mathrm{BaCl}_{2}$ solutions at different driving force

The following cations $\left(\mathrm{Na}^{+}, \mathrm{K}^{+}, \mathrm{Zn}^{2+}, \mathrm{Al}^{3+}, \mathrm{Cu}^{+}\right)$ are shown to have quite a dramatic impact on the $c$-axis length when crystallized at $75^{\circ} \mathrm{C}$ (see Table 2). Notably, the effect is not limited to $1+$ cations, with both zinc and aluminium ions showing a $c$-axis lengthening when compared to the same supersaturation. At high temperatures, where kinetics dominate, many cations are shown to be promoters of $c$-axis growth. The fact that this promotion is, in some cases, only observed at high temperature (eg. $\mathrm{Zn}^{2+}$, unpublished results) suggests that this promotion is indeed kinetically driven.
Table 2 Barium sulfate particles crystallized at $75{ }^{\circ} \mathrm{C}$ from $\mathrm{Na}_{2} \mathrm{SO}_{4}$ and $\mathrm{BaCl}_{2}$ solutions at different driving force in the presence of different cations

Water structure effects also cannot explain the effect of $\mathrm{Zn}^{2+}$ since this ion would be expected to be strongly hydrated (kosmotrope), nor can its impact be predicted by its solubility. Thus, we believe these to be impacting on the surface in such a way as to lower the activation energy barrier of a rate determining step. It is possible that these ions stabilize the step growths where barium ions may incorporate (as per Figure 2a) or it is also possible that these ions form weakly bound ion-pairs with sulfate on the barium sulfate surface and that barium ions are able to replace these cations in this weakly bound structure.

\section{Organic Promoters}

As mentioned in the introductory paragraphs, there is a great deal of literature looking at the crystallization promotion of organic crystals by other organic molecules (eg the work of Weissbuch in ref ${ }^{11}$ ). In this section, we will focus on the promotion of inorganic crystals by organic molecules. In this field, the focus is on the biomineralization of calcite by biomolecules (proteins, amino acids, etc.) and as would be expected from living biological organisms, there is no simple answer as to the impact such mixtures of organic components have on calcite crystallization. Kim et al. ${ }^{26}$ noted on the protein AP7 that "AP7 itself may perform more than one task within the nacre mineralization process" selectively inhibiting some faces while promoting others. In many biomineralization phenomena the initial solid precipitate is amorphous as opposed to crystalline and this may be an important step in biomineralization generally. That is, the kinetic product is formed first. This is also the case for the PILP (polymer induced liquid precursor) process (see below for more on this phenomenon). ${ }^{27}$ In the initial stages, when the carboxylate-rich polymer comes into contact with the calcium ions, the most recent literature tells us that the polymer induces ACC (amorphous calcium carbonate) precipitation. 
Only with time does the initially formed ACC then transform into a crystalline product.

However, while these processes appear to be thermodynamic and result in stabilization of kinetic products, there is also evidence that organic molecules can affect the kinetics of step growth and thereby result in overall crystallization promotion ${ }^{10,28}$. The growth of steps can be given by the step velocity, which (in the case of calcium carbonate crystallization) follows:

$\mathrm{v}=\omega \beta\left(\mathrm{a}_{\mathrm{Ca} 2+}-\mathrm{a}_{\mathrm{Ca} 2+\mathrm{e}}\right)$

where $\mathrm{v}=$ step velocity, $\mathrm{a}=$ activities, $\omega$ is molecular volume and $\beta$ the kinetic co-efficient. The kinetic co-efficient is related to the density of kink sites and to the attachment probability at those sites. The analysis for calcium carbonate shows that these molecules impact on the kinetic co-efficient by lowering the kinetic energy barrier to attach ions to the kink sites. The proposed mechanism hypothesises that this process is able aid in the dehydration of the cation. In addition, in most cases, it is observed that the promotion occurs only at low concentrations (see Figure 5).

Fig. 5 Step growth rate in the presence of polyaspartates showing growth rate promotion at low concentrations followed by inhibition. (Reproduced with permission from Cryst. Growth Des., 2006, 6, 197. Copyright 2006 American Chemical Society). ${ }^{10}$

Recently, molecular dynamics has shown that the small aspartate molecule can promote barium sulfate crystallization. ${ }^{24}$ It appears that some binding of the organic with the cation is desirable and can lead to at least partial dewatering of the cation, a known kinetic barrier for (001) barium sulfate crystallization. ${ }^{23}$ The binding between organic and cation needs to also be weak enough for the cation to be subsequently released, hopefully near the surface where it can incorporate into the growing crystal (see Figure 6). At higher concentrations, thermodynamic effects, such as adsorption of the aspartate at growth sites, occurs and inhibition results. This effect has also been observed for calcium carbonate as discussed above. Thus as concentration increases the equilibrium favours the adsorption process and the thermodynamic effects dominate, while at low concentrations, the kinetic effects dominate. This highlights some of the difficulty associated with a thorough investigation of crystallization mechanisms.

Hydrophilicity is an important parameter since, as discussed for aspartate, if the binding of the polymer to the surface is too strong, inhibition of crystallization will result. Thus, a fine balance between the hydrophobic nature of the organic acid and the concentration (where little adsorption is expected to occur) can result in crystallization promotion of calcium carbonate or barite.

Fig. 6 Mechanism of $\mathrm{Ba}^{2+}$ adsorption in the presence of aspartic acid. Water molecules are shown as grey spheres, hydrogen atoms are not shown for clarity. a) Crystal surface after equilibration showing the ordered first hydration layer; b) At the beginning of the simulation the aspartic acid spontaneously coordinates the $\mathrm{Ba}^{2+}$ ion; c) the aspartic acid molecule assists the $\mathrm{Ba}^{2+}$ permeation through the water layer; d) at the end of the simulation the $\mathrm{Ba}^{2+}$ ion is on the surface, weakly coordinated by the aspartic acid. In $\mathrm{b}, \mathrm{c}$ and $\mathrm{d}$ only the waters of the $\mathrm{Ba}^{2+}$ first hydration shell are shown. (Reproduced from CrystEngComm, 2007, 9, 1187 with permission of the Royal Society of Chemistry). ${ }^{24}$

More recently, polyLysine has also been shown to promote calcium carbonate crystallization ${ }^{29}$ once again with the caveat that low concentrations are present. However, rather than a kinetic mechanism being proposed, it was suggested this was due to non-specific binding. However, no detail on how binding (or adsorption) promotes crystallization is given.

\section{Crystal Growth Modifier - Crystal Hybrids}

The work described above focuses on how a crystal modifier alters the thermodynamic and kinetic processes involved in crystal growth, predominantly at or near the crystal surface. Modifiers may also be incorporated into the crystal structure with widely varying impacts on the crystal growth process and the nature of the final product. ${ }^{30-32}$ The ability of the impurity to become part of the growing crystal, of course, has direct implications for biomineralization where many structures involve the intimate 
association of organic matrix to inorganic crystallizing phase. In fact, the formation mechanisms of these hybrids are thought to be among the possible mechanisms for biomineralization.

Dye inclusion crystals (DICs) are beautiful examples of additive-crystal hybrid materials where the host crystal structures are only slightly perturbed. It is well established that growing crystals can orient and overgrow particular coloured or luminescent additives. When this occurs selectively, the crystal is coloured in specific zones leading to a range of different motifs, including hourglass and Maltese cross inclusions (Figure 7) The comprehensive review by Kahr and Gurney reveals the broad range of known DICs. ${ }^{33}$ While the surface adsorption of dye molecules does have an impact on the crystal growth rate and morphology, ${ }^{34}$ the overgrowth and inclusion of these relatively large organic molecules into the lattice of simple ionic salts such as potassium sulfate, results in essentially unchanged crystal structures. ${ }^{35}$ Recent work has shown that dyes can be selectively incorporated at distinct kink sites, with analysis of the resulting dyed crystals providing insights into the crystal growth mechanism. ${ }^{36}$ In addition, DICs have potential applications in non-linear optical devices and solid-state dye lasers.

Fig. 7 A Maltese Cross inclusion of $\mathrm{K}_{2} \mathrm{SO}_{4}$ dyed with Evan's blue, and an hourglass inclusion of sulforhodamine $\mathrm{B}$, also in $\mathrm{K}_{2} \mathrm{SO}_{4}$ (Reproduced with permission from Chem. Rev., 2001, 101, 893. Copyright 2001 American Chemical Society). ${ }^{33}$

In contrast to DICs, the structures termed mesocrystals are examples of crystalline structures that are significantly different to the unmodified crystal. Cölfen's group has described this phenomenon in detail for calcite, modified by block copolymers. ${ }^{37}$ The resulting crystal growth mechanism is shown schematically in Figure 8 , in comparison with the classical process. A number of recent reviews of these phenomena are available, and we provide only a brief overview here. ${ }^{3,4,31,32,38}$ Whereas the classical model of crystallization involves the amplification of small particles initially formed from solution, non-classical crystallization mechanisms are based on the aggregation of nanoparticles. Oriented attachment of the nanoparticles leads to a structure that can fuse to form a single crystal. If the surfaces of the nanoparticle are sufficiently stabilized, the resulting mesocrystal persists. It is important to note that, due to the alignment of the nanocrystals, a mesocrystal scatters similarly to a single crystal, making detection of these structures non-trivial. In many cases, mesocrystals may not be recognized as such and it seems likely that this area of crystal growth will continue to expand rapidly as more examples are discovered. It is also significant that mesocrystals have been found in the absence of stabilizing additives. ${ }^{3,38}$ Most reported examples of mesocrystalline structures involve inorganic materials, including the widely studied calcite, various oxides, and other sparingly soluble salts. Mesocrystals of DL-alanine are a notable example of an organic system, indicating that this phenomenon can occur in a wide range of systems. ${ }^{39}$ Returning to calcium carbonate, Cölfen et al have recently shown how systematically changing the additive and solute concentrations can give rise to a wide range of morphologies through nonclassical crystallisation. ${ }^{40}$

Fig. 8 Schematic representation of classical and nonclassical crystallization. Pathway (a) the classical crystallization pathway where nucleation clusters grow to a primary nanoparticle, which is amplified to a single crystal. Pathway (b) the primary nanoparticles form an isooriented crystal, which can fuse to form a single crystal. Pathway (c) the primary nanoparticles can be stabilized by an additive and can form a mesocrystal. Pathway (d) Amorphous particles or liquid droplets are formed, which can transform to complicated morphologies. Reproduced from reference 4 with permission of John Wiley \& Sons.

Underpinning the work on oriented attachment crystal growth is an increasing body of knowledge regarding the prenucleation behaviour of well studied systems. The formation of thermodynamically stable prenucleation clusters has been demonstrated for calcite, calcium phosphate and calcium oxalate, which contrasts with the classical view of the process which invokes metastable cluster formation under kinetic control. ${ }^{41}$ Introducing 
additives into these systems can then result in a range of different outcomes, the complexity of which is perhaps best illustrated in the recent work of Cölfen et al where up to nine independent actions of an additive upon a crystallizing system are described. ${ }^{42}$

Another aspect of these non-classical crystal growth mechanisms is the formation of liquid phase precursors, as shown in Figure 8. In many of the reported instances of this phenomenon, the liquid precursors are stabilized by a polymeric additive, leading to the term "polymer induced liquid precursor" (PILP) introduced by Gower and Tirrell, as mentioned above. ${ }^{27,43}$ This is particularly significant as the liquid can be controlled to form an infinite array of shapes and structures. Subsequent crystallization leads to crystalline morphologies that are difficult or impossible to achieve by other means. The underlying mechanism is under active investigation, but it is notable that liquid-like precursors have been reported in the absence of additives, suggesting that the polymer additives are stabilizing an otherwise unstable stage of the crystallization process. ${ }^{27}$ Initially reported for calcium carbonate, PILPs have been reported in other inorganic systems, and to a lesser extent, organic compounds, ${ }^{27,38}$ including a very recent report where this mechanism is applied to an industrial organic pigment. ${ }^{44}$

\section{Conclusions}

Overall, it is clear that the study of crystal growth is a vibrant area of research that we have only been able to cover briefly here. It is fascinating to consider what a small percentage of known crystalline systems have been studied in any detail from a crystal growth perspective. Metal-organic frameworks (MOFs) are examples of systems that have been intensively studied in recent times, but studies of the crystal growth mechanisms and associated phenomena are relatively few to date. ${ }^{45} \mathrm{We}$ hope that the recent developments discussed here, which are being driven largely by interest in biomineralization and associated systems, will be considered in the study of the full tapestry of crystalline materials.

\section{Notes and references}

${ }^{a}$ Department of Chemistry, Curtin University of Technology, GPO Box U 1987, Perth, Western Australia, 6845. Fax:+618 9266 4699; Tel:+618 9266 2483; E-mail: f.jones@curtin.edu.au, m.ogden@,curtin.edu.au

1. J.Y. Lee, B.H. Hong, W.Y. Kim, S.K. Min, Y. Kim, M.V. Jouravlev, R. Bose, K.S. Kim, I.C. Hwang, L.J. Kaufman, C.W. Wong, P. Kim and K.S. Kim, Nature, 2009, 460, 498.; X.L. Fang, C. Chen, M.S. Jin, Q. Kuang, Z.X. Xie, S.Y. Xie, R.B. Huang and L.S. Zheng, J. Mater. Chem., 2009, 19, 6154.

2. H. Colfen, Top. Curr. Chem., 2007, 271, 1.; S.H. Yu, Top. Curr. Chem., 2007, 271, 79.

3. A.W. Xu, Y.R. Ma and H. Cölfen, $J$. Mater. Chem., 2007, 17, 415.

4. H. Cölfen and M. Antonietti, Mesocrystals and Non Classical Crystallization, John Wiley \& Sons, Chichester, U.K, 2008.

5. F. Wang, G.Y. Xu, Z.Q. Zhang, S. Song and S.L. Dong, J. Colloid Interf. Sci., 2006, 293, 394.

6. S. Garnier, S. Petit and G. Coquerel, $J$. Cryst. Growth, 2002, 234, 207.

7. E. Ruiz-Agudo, C.V. Putnis and C. Rodriguez-Navarro, Cryst. Growth Des., 2008, 8, 2665.

8. F. Jones, W.R. Richmond and A.L. Rohl, J. Phys. Chem. B, 2006, 110, 7414.

9. A.L. Rohl, D.H. Gay, R.J. Davey and C.R.A. Catlow, J. Am. Chem. Soc., 1996, 118, 642.

10. S. Elhadj, E.A. Salter, A. Wierzbicki, J.J. De Yoreo, N. Han and P.M. Dove, Cryst. Growth Des., 2006, 6, 197.

11. R. Popovitz-Biro, I. Weissbuch, D. Jacquemain, F. Leveiller, L. Leiserowtiz and M. Lahav, in "Advances in Industrial Crystallization”, p 3-19, J. Garside, R.J. Davey and A.G. Jones Eds., Butterworth-Heinemann Ltd, 1991.; I. Weissbuch, L. Leiserovitz and M. Lahav, in "Crystallization Technology Handbook", p 563-586, A. Mersmann Ed., Marcel-Dekker Inc, 1995. 
12. D. Volkmer, M. Fricke, C. Agena and J. Mattay, J. Mater. Chem., 2004, 14, 2249.

13. J. Amsler, Helv. Phys. Acta, 1942, 699.

14. A.K. Kalia, J. Cryst. Growth, 1976, 35, 334.

15. S.T. Liu, G.H. Nancollas and E.A. Gasiecki, J. Cryst. Growth, 1976, 33, 11.

16. S.L. HE, J.E. ODDO and M.B. TOMSON, J. Colloid Interface Sci., 1995, 174, 319.

17. P. Risthaus, D. Bosbach, U. Becker and A. Putnis, Colloids Surf. A, 2001, 191, 201.

18. M. Kowacz, C. Putnis and A. Putnis, Geochim. Cosmochim. Acta, 2007, 71, 5168.

19. U. Becker, P. Risthaus, D. Bosbach and A. Putnis, Mol. Simulat., 2002, 28, 607.

20. M. Kowacz and A. Putnis, Geochim. Cosmochim. Acta, 2008, 72, 4476.

21. C.M. Pina, M. Enders and A. Putnis, Chem. Geol., 2000, 168, 195.

22. F. Jones, S. Piana and J.D. Gale, Cryst Growth Des, $2008,8,817$.

23. S. Piana, F. Jones and J.D. Gale, J. Am. Chem. Soc., 2006, 128, 13568.

24. S. Piana, F. Jones and J.D. Gale, CrystEngComm, 2007, 9, 1187.

25. S.W. Benson, Thermodynamical Kinetics $2^{\text {nd }}$ Edition, John Wiley, New York, USA, 1976.

26. I.W. Kim, S. Collino, D.E. Morse and J.S. Evans, Cryst. Growth Des., 2006, 6, 1078.

27. L.B. Gower, Chem. Rev., 2008, 108, 4551 .

28. I.W. Kim, M.R. Darragh, C. Orme and J.S. Evans, Cryst. Growth Des., 2006, 6, 5.; A.E. Stephenson, J.J. DeYoreo, L. Wu, K.J. Wu, J. Hoyer and P.M. Dove, Science, 2008, 322, 724.

29. B. Njegic-Dzakula, L. Brecevic, G. Falini and D. Kralj, Cryst. Growth Des., 2009, 9, 2425.

30. L.J. Wang and G.H. Nancollas, Chem. Rev., 2008, 108, 4628.
31. L.J. Wang and G.H. Nancollas, Dalton Trans., 2009, 2665.

32. F.C. Meldrum and H. Cölfen, Chem. Rev., 2008, 108, 4332.

33. B. Kahr and R.W. Gurney, Chem. Rev., 2001, 101, 893.

34. D.J. Carter, M.I. Ogden and A.L. Rohl, J. Phys. Chem. C, 2007, 111, 9283.

35. W.M. Vetter, H. Totsuka, M. Dudley and B. Kahr, J. Cryst. Growth, 2002, 241, 498.

36. T. Bullard, K.L. Wustholz, E.D. Bott, M. Robertson, P.J. Reid and B. Kahr, Cryst. Growth Des., 2009, 9, 982.

37. A.N. Kulak, P. Iddon, Y.T. Li, S.P. Armes, H. Cölfen, O. Paris, R.M. Wilson and F.C. Meldrum, J. Am. Chem. Soc., 2007, 129, 3729 .

38. Q. Zhang, S.J. Liu and S.H. Yu, J. Mater. Chem., 2009, 19, 191.

39. S. Wohlrab, N. Pinna, M. Antonietti and H. Cölfen, Chem. Eur. J., 2005, 11, 2903.

40. R.Q. Song, H. Cölfen, A.W. Xu, J. Hartmann and M. Antonietti, ACS Nano, 2009, 3, 1966.

41. D. Gebauer, A. Volkel and H. Cölfen, Science, 2008, 322, 1819.

42. D. Gebauer, H. Cölfen, A. Verch and M. Antonietti, Adv. Mater., 2009, 21, 435.

43. L.A. Gower and D.A. Tirrell, J. Cryst. Growth, 1998, 191, 153.

44. Y.R. Ma, G. Mehltretter, C. Plug, N. Rademacher, M.U. Schmidt and H. Cölfen, $A d v$. Funct. Mater., 2009, 19, 2095.

45. R.E. Morris, ChemPhysChem, 2009, 10, 327. 


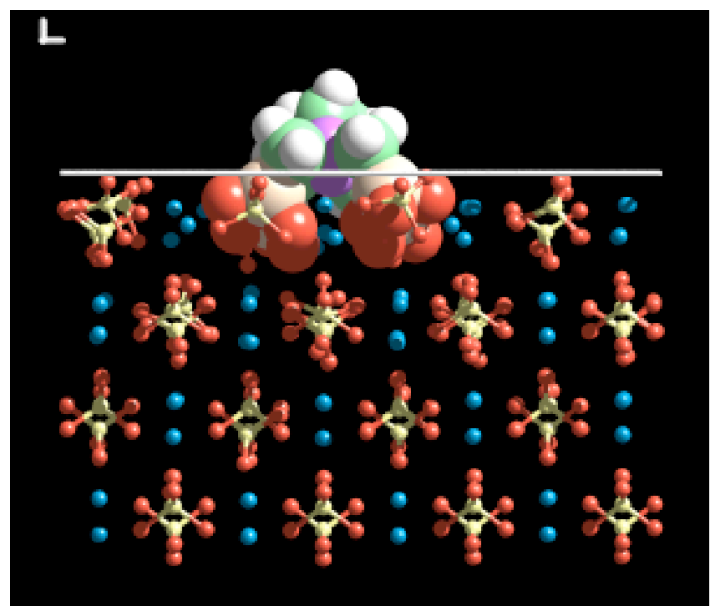

Figure 1.

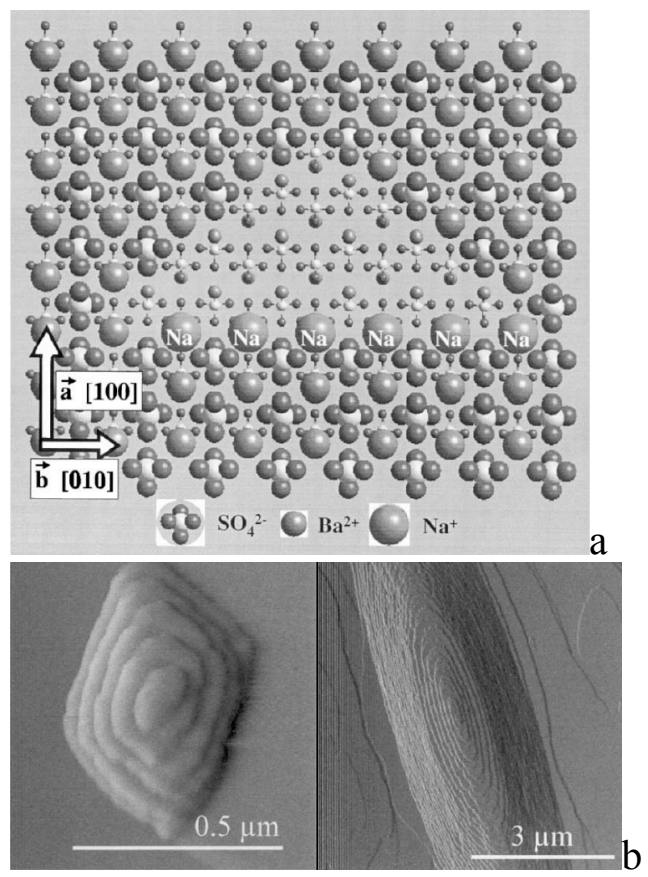

Figure 2.

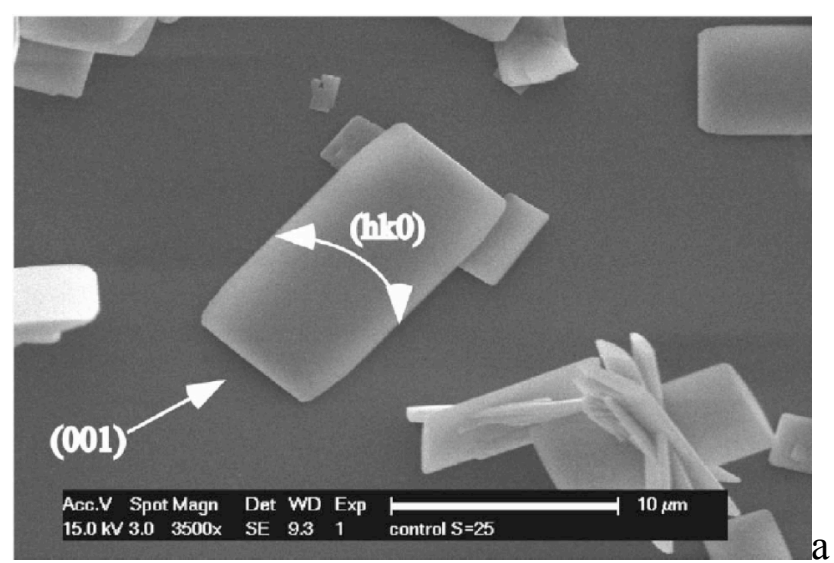

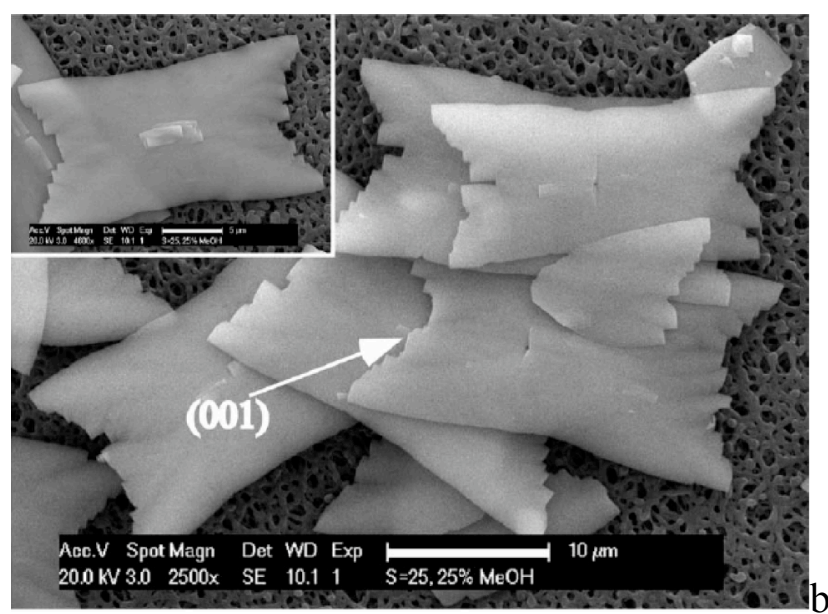

Figure 3.

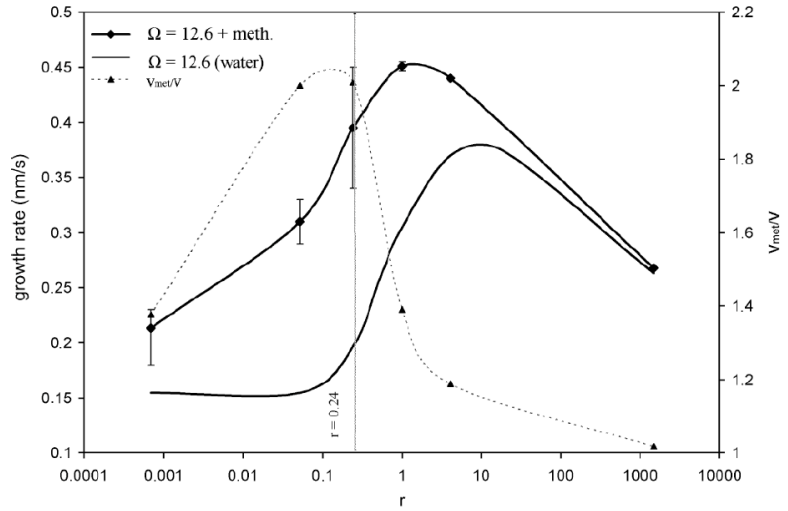

Figure 4.

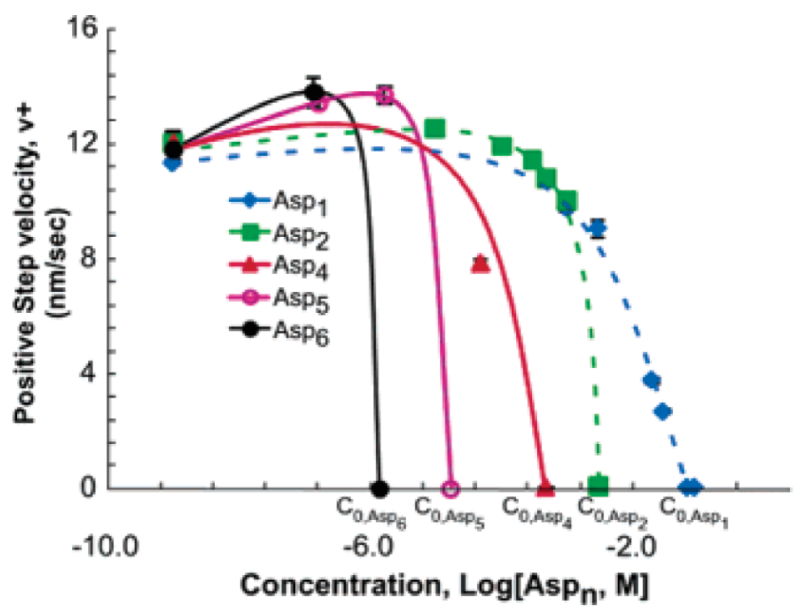

Figure 5. 


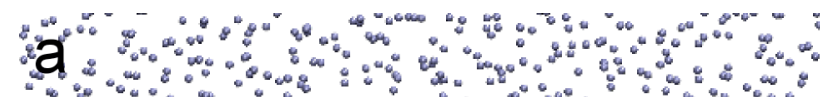
4. \& ०० $4060 \%$ to

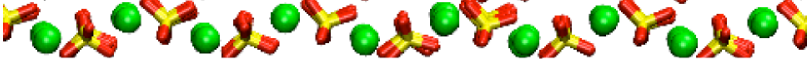

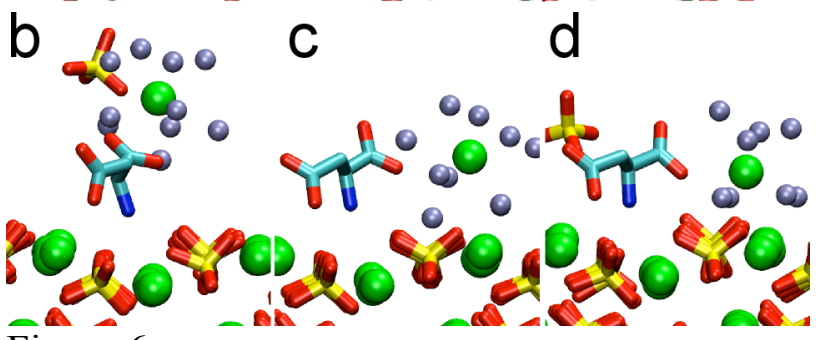
Figure 6.
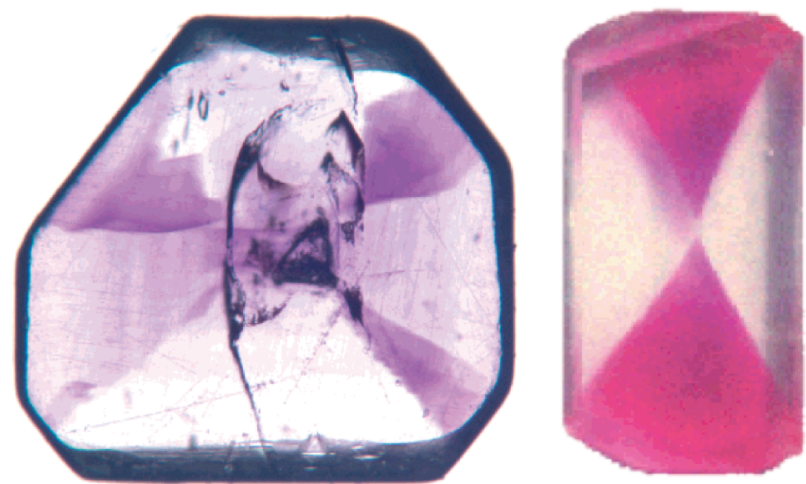

Figure 7.

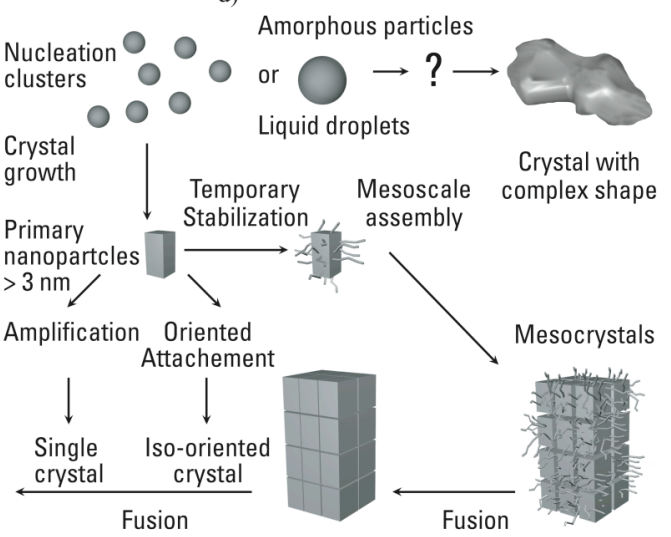

c)

Figure 8. 